\title{
A Modular Synthesis of Conformationally Preorganised Extended $\beta$-Strand Peptidomimetics
}

\author{
Tohru Yamashita, Peter C. Knipe, Nathalie Busschaert, Sam Thompson* and Andrew D. Hamilton*
}

\begin{abstract}
A promising strategy for mediating protein-protein interactions is the use of non-peptidic mimics of secondary structural protein elements such as the $\alpha$-helix. Recent work has expanded this toolkit by providing proof-of-principle scaffolds that are conformationally biased to mimic the projection of side-chains from one face of another common secondary structural element - the $\beta$ strand. Herein we present a synthetic route that has key advantages over previous work: monomers bearing an amino acid side-chain are preformed before rapid assembly to peptidomimetics via a modular, iterative strategy. The resultant oligomers of alternating pyridyl and six-membered cyclic ureas accurately reproduce a recognition domain of several amino acid residues of a $\beta$-strand - demonstrated here by mimicry of the $i, i+2, i+4$ and $i+6$ residues.
\end{abstract}

The inhibition or stabilization of protein-protein interactions (PPIs) represents a potential goldmine for medicinal chemistry, ${ }^{[1]}$ yet the rational design of synthetic agents able to achieve this goal has been seen by many as intractable until recently. ${ }^{[2]}$ There is growing interest in the synthesis of non-peptidic mimics of secondary ${ }^{[3]}$ and supersecondary ${ }^{[4]}$ protein elements and the side-chain residues they present for the recognition of cognate partners. There has been considerable success in mimicking the $\alpha$-helical structures that are ubiquitous at interfacial peptide regions, prompting researchers to develop scaffolds for the projection of groups that reproduce side-chain vectors from one,${ }^{[5]}$ or two, ${ }^{[6]}$ faces of the helix.

PPIs mediated by extended regions of proteins such as $\beta$-strands (Figure $1 \mathrm{~A})^{[7]}$ are also of great interest, with our group ${ }^{[8]}$ and others, ${ }^{[9]}$ providing strategies towards minimalist frameworks that replicate their key domains (Figure 1B).

Dr. P. C. Knipe, Dr. T. Yamashita, Dr. N. Busschaert, Dr. S. Thompson, Prof. Dr. A. D. Hamilton

Chemistry Research Laboratory, University of Oxford

12 Mansfield Road, Oxford, OX1 3TA (UK)

E-mail: sam.thompson@chem.ox.ac.uk andrew.hamilton@chem.ox.ac.uk

Homepage: http://hamilton.chem.ox.ac.uk

Abbreviations \& acronyms: $\mathrm{Bn}=$ benzyl; Boc = tert-butoxycarbonyl; $\mathrm{dba}=$ dibenzylideneacetone; DIBAL-H = diisobutylaluminium hydride; $\mathrm{DMF}=$ $\mathrm{N}, \mathrm{N}$-dimethylformamide; $\mathrm{MM}=$ molecular mechanics; Ms, mesyl = methanesulfonyl; NOESY = nuclear Overhauser effect spectroscopy; RMSD = root-mean-square deviation; ROESY = rotating frame NOESY; Ts, tosyl = para-toluenesulfonyl; Xantphos $=4,5$-bis(diphenylphosphino)9,9-dimethylxanthene.

Supporting information for this article is given via a link at the end of the document.

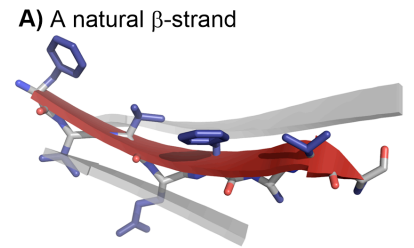

B) Schematic representation
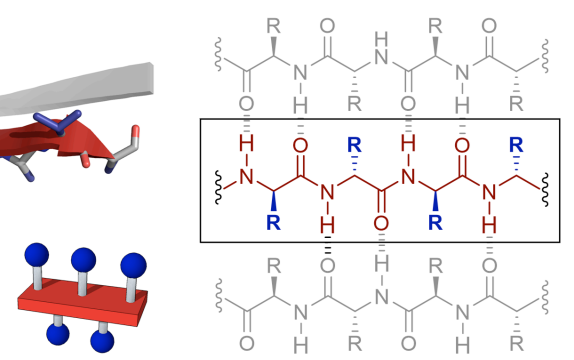

C) Previous work: pyridyl-imidazolidinone foldamers as dipole-stabilized mimics

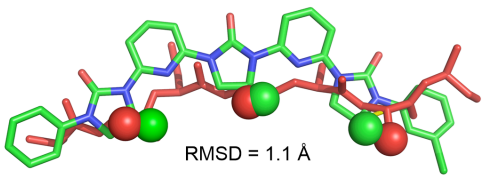<smiles></smiles>

D) This work: a novel pyridyl-tetrahydropyrimidone scaffold

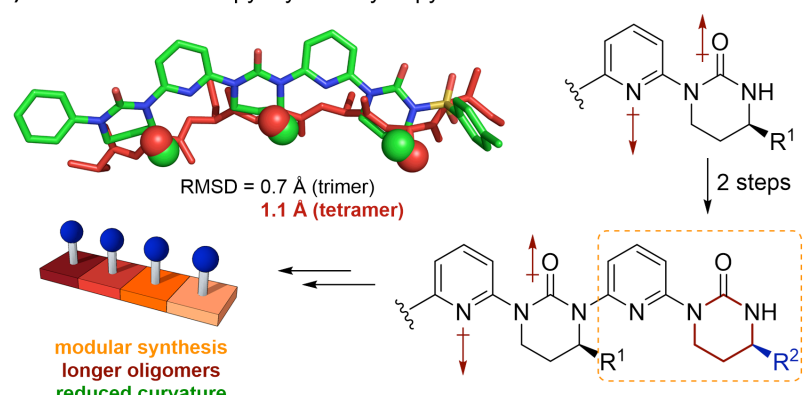

Figure 1. $\beta$-Strand peptidomimicry; A) canonical $\beta$-strand; B) schematic of side-chain projection; C) previous work: overlay of a three-residue mimic rigidified through dipolar repulsion (green) with a strand (red); D) this work: a modular synthesis from preformed monomers provides rapid access to foldamers of alternating six-membered rings. Increased linearity over extended distances allows mimicry of longer peptide sequences. For example the sixpoint (trimer) RMSD is reduced to $0.7 \AA$, and the eight-point value for the tetramer is $1.1 \AA$. $\beta$-Strand from PDB: $3 Q X T$, side-chains represented as spheres for clarity, $\alpha$ - and $\beta$-carbons used for calculation. ${ }^{[12]}$

We recently described a homochiral foldamer of alternating 2,6-disubstituted pyridyl groups linked by imidazolidin-2-ones bearing methyl groups, resulting in the mimicry of side-chains from the $i, i+2$, and $i+4$ positions of one face of a $\beta$-strand. ${ }^{[10]}$ An important conformational feature of this motif is preorganization of the scaffold by dipolar repulsion between the pyridyl nitrogen lone pair and the carbonyl groups of adjacent imidazolidinones. The resultant projection of three groups for side-chain mimicry is in good agreement with those of one face of a canonical strand (Figure 1C).

Extending the scope of this design for the mimicry of amino acid sequences over longer stretches of peptide requires two challenges to be overcome: (i) the alternation of five- and six-membered rings introduces a backbone curvature that is 
increasingly divergent from that of a canonical strand; (ii) a linear synthesis in which each side-chain mimic is formed on the growing oligomeric strand is inherently low-yielding. A potential solution is to pre-form six-membered cyclic urea monomers that mimic a given amino acid side-chain prior to oligomerization. As one-carbon homologues of the imidazolidin-2-one, the resultant foldamer is much more linear - thus more closely reproducing the side-chain vectors of a $\beta$-strand. ${ }^{[11]}$ Calculation suggests a reduction in six-point RMSD value from $1.1 \AA$ to $0.7 \AA$ for the five- and six-membered urea mimics, respectively. ${ }^{[12 a, b]}$ Preforming each monomer has the potential to drastically reduce the longest linear synthetic sequence and more readily allows a peptidomimic of several residues to be varied at a particular position and generated in an iterative fashion (Figure 1D). There are numerous commercially available derivatives of enantiomerically pure $\alpha$-amino alcohols bearing a wide variety of side-chains, making them an ideal starting material for monomer construction.

As a representative amino acid found commonly in $\beta$-strands we chose to explore a proof-of-principle strategy based on phenylalanine. One-carbon homologation of $N$-Boc-Lphenylalaninol 1 via $\mathrm{O}$-mesylation and displacement with sodium cyanide afforded nitrile 2 , which was transformed to the aniline derivative 3 via DIBAL-H mediated reduction followed by reductive amination with sodium cyanoborohydride and aniline. Trifluoroacetic acid mediated removal of the Boc group and cyclization with triphosgene gave terminally capped monomer 4 (Scheme 1A).

$$
\text { A) }
$$

Scheme 1. The synthesis of monomeric cyclic ureas bearing an amino acid side-chain mimic for: A) uni-directional functionalization; B) bi-directional functionalization. Reagents and conditions: (a, i) $\mathrm{MsCl}$ (1.2 eq.), $\mathrm{Et}_{3} \mathrm{~N}$ (1.5 eq.), $\mathrm{CH}_{2} \mathrm{Cl}_{2}, 0{ }^{\circ} \mathrm{C} \rightarrow \mathrm{rt}, 10$ mins; (ii) $\mathrm{NaCN}$ (2.5 eq.), DMF, $60{ }^{\circ} \mathrm{C}, 3 \mathrm{~h}, 66 \%$ over two steps, (b, i) DIBAL-H (2.5 eq.), $\mathrm{CH}_{2} \mathrm{Cl}_{2},-78{ }^{\circ} \mathrm{C} \rightarrow 0{ }^{\circ} \mathrm{C}, 1 \mathrm{~h}$; (ii) aniline (2 eq.) $\mathrm{NaBH}_{3} \mathrm{CN}$ (3 eq.), AcOH, $\mathrm{MeOH}$, rt, $16 \mathrm{~h}, 19 \%$ over two steps, (c, i) trifluoroacetic acid, $\mathrm{CH}_{2} \mathrm{Cl}_{2}, \mathrm{rt}, 0.5 \mathrm{~h}$; (ii) triphosgene $\left(0.5\right.$ eq.), $\mathrm{iPr}_{2} \mathrm{NEt}$ (4 eq.), rt, $1 \mathrm{~h}, 47 \%$ over two steps, (d) $\mathrm{NaCN}$ (1.35 eq.), $\mathrm{MeCN}, \mathrm{H}_{2} \mathrm{O}$, reflux, $1.5 \mathrm{~h}, 84 \%$, (e, i) $\mathrm{NiCl}_{2}$ (2 eq.), $\mathrm{NaBH}_{4}$ (10 eq., portion-wise), $\mathrm{MeOH}, 0{ }^{\circ} \mathrm{C} \rightarrow \mathrm{rt}$, 10 mins; (ii) triphosgene ( 0.5 eq.), $\mathrm{Pr}_{2} \mathrm{NEt}$ ( 2 eq.), rt, $2 \mathrm{~h}, 47 \%$ over two steps, (f) 2,6-dibromopyridine (2 eq.), XantPhos (30 mol\%), $\mathrm{Pd}_{2} \mathrm{dba}_{3}$ (10 $\mathrm{mol} \%$ ), $\mathrm{CsCO}_{3}$ (2 eq.), dioxane, $80{ }^{\circ} \mathrm{C}, 1.5 \mathrm{~h}, 54 \%$

The iterative monomer $\mathbf{8}$ was synthesized from commercially available aziridine $\mathbf{5}$. Opening of the threemembered ring with sodium cyanide gave $\mathbf{6}$, followed by nickel boride-mediated reduction and cyclization with triphosgene to produce 7 in $40 \%$ overall yield. Subsequent Buchwald-Hartwig amination of 2,6-dibromopyridine with 7 gave tosyl-protected monomer 8 in $54 \%$ yield (Scheme 1B).

Monomers $\mathbf{4}$ and $\mathbf{8}$ were coupled under Buchwald-Hartwig conditions to give two-residue mimic 9 in an excellent yield of
$96 \%$. The oligomer can be rapidly extended to a mimic of three11 and four-residues 13 via an iterative two-step sequence of $\mathrm{N}$-tosyl deprotection with magnesium in methanol followed by Buchwald-Hartwig cross-coupling with bromide 8 (Scheme 2).

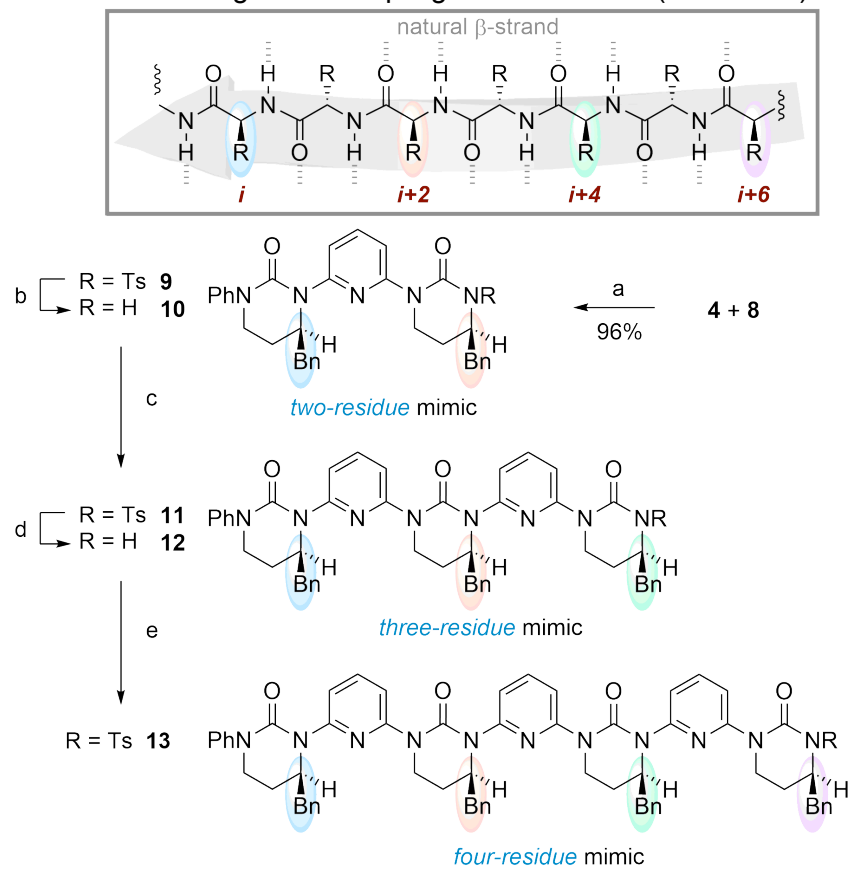

Scheme 2. Oligomer homologation to form conformationally rigidified peptidomimetics of two $(9,10)$, three $(11,12)$ and four $(13)$ side-chain residues from one face of a natural $\beta$-strand. Reagents and conditions: (a) condition A, 96\%; (b) condition B, 56\%; (c) condition A, 72\%; (d) condition B, 10\%; (e) condition A, 52\%. Condition A: XantPhos (30 mol\%), $\mathrm{Pd}_{2} \mathrm{dba}_{3}(10 \mathrm{~mol} \%$ ), $\mathrm{CsCO}_{3}$ (2 eq.), dioxane, $90{ }^{\circ} \mathrm{C}, 16 \mathrm{~h}$; condition B: $\mathrm{Mg}(25$ eq. $), \mathrm{MeOH}$, sonication, rt, $10 \mathrm{~min}$.

Single crystals suitable for X-ray diffraction were obtained for monomer $\mathbf{8} .^{[13]}$ The solid-state conformation is consistent with dipolar repulsion between the urea carbonyl group and the pyridine nitrogen atom favoring an anti- relationship (Figure $2 \mathrm{~A}$ ). The largely planar cyclic urea adopts a puckered conformation placing the benzyl group in a pseudo-axial position (Figure 2B, Supporting Information). ${ }^{[14]}$ To probe the preferences of the higher order homologues 9-13 we conducted a computational search of their lowest energy conformations. ${ }^{[12 b, c]}$ The validity of this method was confirmed by the close agreement between the solid-state structure of monomer 8 and the lowest energy conformer by calculation (RMSD $0.5 \AA$, see Supporting Information). Calculations for mimics 8-13 gave lowest energy structures adopting fully extended conformations with urea carbonyl groups anti- to pyridine nitrogens, and with R-groups in pseudo-axial positions (Supporting Information). Consequently the phenylalanine side-chain mimics are projected from a common face, and thus in good agreement with those of a canonical $\beta$-strand. Trimer 11 mimics the $i, i+2, i+4$ residues with a six-point RMSD value of $0.7 \AA$, while tetramer 13 adds the $i+6$ residue and has an eight-point RMSD value of $1.1 \AA$ (Scheme 2, Supporting Information). ${ }^{[12 a]}$ 
A)

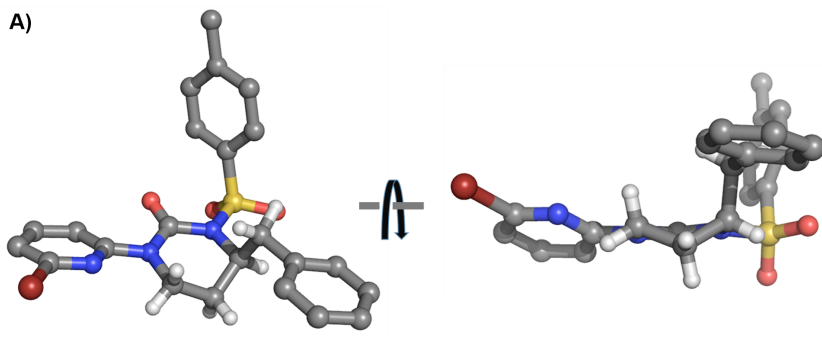

B)

axial-Bn
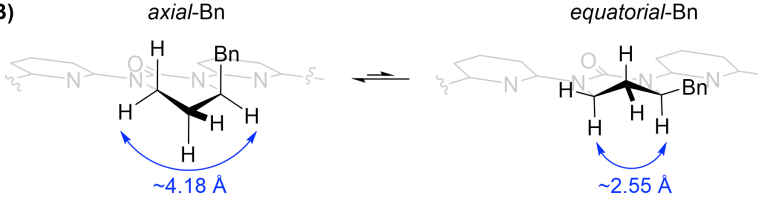

Figure 2. Conformational behaviour of $\beta$-strand mimics 8-13: A) X-ray crystal structure of monomer $\mathbf{8}$, showing the benzyl substituent in a pseudo-axial position; B) equilibrium between ring conformers placing the $\mathrm{Bn}$ side-chain mimic in pseudo-axial and pseudo-equatorial positions. Selected interhydrogen distances given for each conformer based on MM calculations. ${ }^{[12 b, c, 14,15]}$ The difference in inter-hydrogen distance allows the conformers to be distinguished by nOe observations.

Solution-phase conformational behavior was probed with NOESY and ROESY NMR experiments in $\mathrm{CDCl}_{3}$ (Figure 3). nOe Signals between the pyridine- and cyclic urea-hydrogens, e.g. $\mathrm{H} 8 \leftrightarrow \mathrm{H} 15$ and $\mathrm{H} 20 \leftrightarrow \mathrm{H} 17$ for dimer 9, were not observed for compounds 8-13 (Figure 3B dashed red arrows). However, strong correlations were observed between urea-hydrogens and the terminal phenyl-/tosyl-hydrogens by way of comparison, e.g. $\mathrm{H} 6_{\text {eq }} \leftrightarrow \mathrm{H} 3$ and $\mathrm{H} 22_{\text {eq }} \leftrightarrow \mathrm{H} 29$ (Figure 3B/D green arrows, Supporting Information). ${ }^{[15 c, 16]}$ This data is consistent with the previously reported five-membered imidazolidin-2-one analogues,${ }^{[10]}$ indicating that the predominant species in solution adopts the desired extended conformation where the urea carbonyl groups are anti- to the pyridine nitrogen atoms analogous to the conformations depicted in Figures 3B and D. This result highlights the utility of dipolar repulsion as a conformational determinant for pre-organisation of peptidomimics.

Further inspection of the NOESY NMR spectra of $\mathbf{8 - 1 3}$ reveals interesting features regarding the position of the phenylalanine mimic. For dimer 9 the absence of trans-annular nOe correlations between $\mathrm{H} 6$ and $\mathrm{H} 8$, and between $\mathrm{H} 2 \mathrm{O}$ and $\mathrm{H} 22$, was consistent with their occupying pseudo-equatorial positions, ${ }^{[15 a]}$ with the side-chain mimic in a pseudo-axial site (Figures $3 \mathrm{C}, \mathrm{D}$ ). Interestingly, the only nOe signal in this region, corresponds to two hydrogens $(\mathrm{H} 8$ and $\mathrm{H} 20)$ located on adjacent cyclic ureas. ${ }^{[15 b]}$ Additional evidence for this ring conformation is given by cross peaks between the cyclic urea- and benzylichydrogens. For example the nOe signal between benzylic $\mathrm{H} 9$ and one of the diastereotopic $\mathrm{H} 6$ hydrogens (Figure 3D green arrows) is much stronger than it is with the $\mathrm{H} 7$ hydrogen ( $49: 1$ by direct integration of cross peaks). Moreover, one of the diastereotopic $\mathrm{H} 7$ hydrogens has no cross peak with the aromatic region, indicative of occupation of a pseudo-axial position. ${ }^{[16]}$
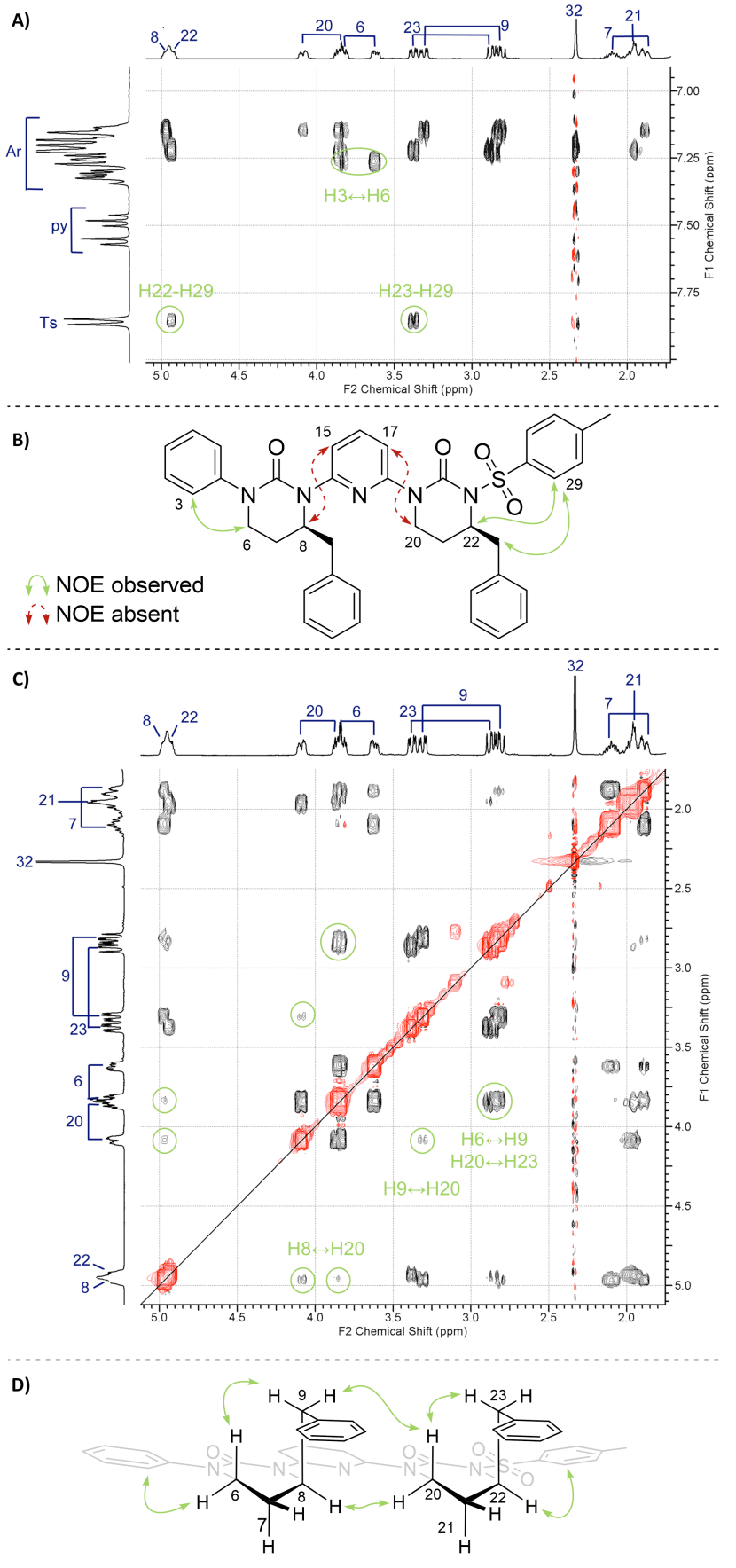

Figure 3. Solution-phase conformational analysis of dimer 9: A) selected region of the NOESY NMR spectrum focusing on cross peaks with the pyridine signals (py); B) conformation based on nOe data; C) selected region of the NOESY NMR spectrum focusing on cross peaks between the cyclic urea hydrogens; D) perspective view of conformation based on nOe data. Key observed (green) and absent (red, dashed) nOe correlations are indicated. $\mathrm{CDCl}_{3}, 400 \mathrm{MHz}, 298 \mathrm{~K}^{[16]}$

In conclusion, we have designed a synthetic route to an oligomer consisting of alternating pyridyl and six-membered cyclic urea groups. The resultant foldamer is conformationally pre-organized by dipolar repulsion and ring puckering to project groups along the same vectors as the side-chains of amino acid 
residues from one face of a $\beta$-strand, as shown by $X$-ray crystallography, NMR and MM calculations. As proof-of-principle we demonstrated that mimics of two, three, and four phenylalanine side-chains gave excellent spatial and angular agreement with those of a canonical strand. A particularly attractive aspect of the approach is that an iterative two-step process allows the foldamer to be extended by incorporation of pre-formed monomers. Work is underway to evaluate the conformational behaviour of mimics of specific peptide sequences containing a range of proteinogenic amino acids, and to assess their efficacy in mediating protein-protein interactions in aqueous media.

\section{Acknowledgements}

We thank The University of Oxford for funding and Dr. Amber L. Thompson for assistance with X-ray crystallography. TY thanks Takeda Pharmaceutical Company Limited for their generosity in providing financial and logistical support during a sabbatical position as Visiting Scientist in The University of Oxford (2014).

Keywords: dipolar repulsion $•$ protein-protein interaction • foldamer • extended conformation $\bullet$ inhibitor

[1] S. Jones, J. M. Thornton, Proc. Natl. Acad. Sci. 1996, 93, 13-20.

[2] a) M. R. Arkin, J. A. Wells, Nat. Rev. Drug Discov. 2004, 3, 301-317; b) M. R. Arkin, Y. Tang, J. A. Wells, Chem. Biol. 2014, 21, 1102-1114

[3] a) M. J. Adler, A. Jamieson, A. Hamilton, in Small-Molecule Inhibitors of Protein-Protein Interactions, Vol. 348 (Eds.: L. Vassilev, D. Fry), Springer Berlin Heidelberg, 2011, pp. 1-23; b) M. K. P. Jayatunga, S. Thompson, A. D. Hamilton, Bioorg. Med. Chem. Lett. 2014, 24, 717 724; c) H. Moon, H.-S. Lim, Curr. Opin. Chem. Biol. 2015, 24, 38-47.

[4] a) O. V. Kulikov, S. Thompson, H. Xu, C. D. Incarvito, R. T. W. Scott, I. Saraogi, L. Nevola, A. D. Hamilton, Eur. J. Org. Chem. 2013, 2013 3433-3445; b) L. Nevola, J. M. Rodriguez, S. Thompson, A. D. Hamilton, Supramol. Chem. 2013, 25, 586-590.

[5] a) V. Azzarito, K. Long, N. S. Murphy, A. J. Wilson, Nat. Chem. 2013, 5, 161-173; b) B. B. Lao, I. Grishagin, H. Mesallati, T. F. Brewer, B. Z. Olenyuk, P. S. Arora, Proc. Natl. Acad. Sci. USA 2014, 111, 75317536; c) G. M. Burslem, H. F. Kyle, A. L. Breeze, T. A. Edwards, A. Nelson, S. L. Warriner, A. J. Wilson, ChemBioChem 2014, 15, 10831087; d) V. Azzarito, J. A. Miles, J. Fisher, T. A. Edwards,
S. L. Warriner, A. J. Wilson, Chem. Sci. 2015, 6, 2434-2443; e) A. Barnard, K. Long, H. L. Martin, J. A. Miles, T. A. Edwards, D. C. Tomlinson, A. Macdonald, A. J. Wilson, Angew. Chem. Int. Ed. 2015, 54, 2960-2965; f) O. V. Kulikov, S. Kumar, M. Magzoub, P. C. Knipe, I. Saraogi, S. Thompson, A. D. Miranker, A. D. Hamilton, Tetrahedron Lett. 2015, 56, 3670-3673.

[6] a) S. Marimganti, M. N. Cheemala, J.-M. Ahn, Org. Lett. 2009, 11, 4418-4421; b) S. Thompson, R. Vallinayagam, M. J. Adler, R. T. W. Scott, A. D. Hamilton, Tetrahedron 2012, 68, 4501-4505; C) S. Thompson, A. D. Hamilton, Org. Biomol. Chem. 2012, 10, 57805782; d) K.-W. Jung, K. Vanommeslaeghe, M. E. Lanning, J. L. Yap, C. Gordon, P. T. Wilder, A. D. MacKerell, S. Fletcher, Org. Lett. 2013, 15, 3234-3237.

[7] a) H. Remaut, G. Waksman, Trends Biochem. Sci. 2006, 31, 436-444; b) A. M. Watkins, P. S. Arora, ACS Chem. Biol. 2014, 9, 1747-1754.

[8] a) P. N. Wyrembak, A. D. Hamilton, J. Am. Chem. Soc. 2009, 131, 4566-4567; b) A. G. Jamieson, D. Russell, A. D. Hamilton, Chem. Commun. 2012, 48, 3709-3711; c) C. L. Sutherell, S. Thompson, R. T. W. Scott, A. D. Hamilton, Chem. Commun. 2012, 48, 9834-9836.

[9] W. A. Loughlin, J. D. A. Tyndall, M. P. Glenn, T. A. Hill, D. P. Fairlie, Chem. Rev. 2010, 110, PR32-PR69.

[10] E. A. German, J. E. Ross, P. C. Knipe, M. F. Don, S. Thompson, A. D. Hamilton, Angew. Chem. Int. Ed. 2015, 54, 2649-2652.

[11] S. W. Fanning, J. R. Horn, Protein Sci. 2011, 20, 1196-1207.

[12] a) For the $C \alpha$ and $C \beta$ atoms of a canonical strand, MMFF94x with Born solvation for chloroform; b) Molecular Operating Environment (MOE), version 2014.09, Chemical Computing Group, Inc., Montreal, QC, Canada, 2014; c) LowModeMD; (see Supporting Information).

[13] CCDC 1056842 (8) contains the supplementary crystallographic data for this paper. These data can be obtained free of charge from The Cambridge Crystallographic Data Centre via www.ccdc.cam.ac.uk/data request/cif.

[14] a) Cremer-Pople puckering coordinates (D. Cremer, J. A. Pople, J. Am. Chem. Soc. 1975, 97, 1354-1358) calculated using PLATON: b) A. L. Spek, Utrecht University, Utrecht, The Netherlands 2001, 20; c) A. L Spek, J. Appl. Crystallogr. 2003, 36, 7-13.

[15] Calculations ${ }^{[12]}$ give inter-nuclear hydrogen distances of: a) $\sim 4.18 \AA$ between 1,3-di-psuedo-equatorial positions; b) $\sim 2.8 \AA$ between pseudoequatorial positions on neighbouring ureas; c) $>4 \AA$ between the pyridine- and urea-hydrogens (see Supporting Information).

[16] ${ }^{1} \mathrm{H}$ NMR and NOESY spectra for dimer 9 were chosen for Figure 3 due to superior resonance dispersion. Observations for trimer 11 and tetramer 13 were consistent with the population of analogous conformational ensembles, see Supporting Information. 


\section{COMMUNICATION}

A promising strategy for mediating protein-protein interactions is the use of non-peptidic mimics of secondary structural protein elements. Here we show that an iterative synthetic route provides access to monomers bearing side-chain mimics that can be rapidly assembled into longer oligomers that adopt well-defined conformations that accurately reproduce the recognition domain of several amino acid residues of a $\beta$-strand.

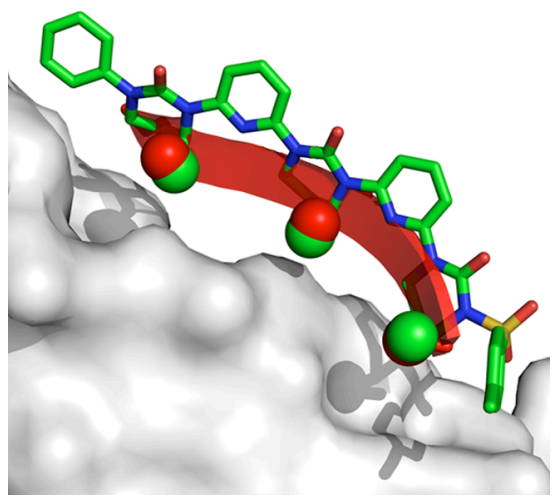

Tohru Yamashita, Peter C. Knipe Nathalie Busschaert, Sam Thompson* and Andrew D. Hamilton*

Page No. - Page No.

A Modular Synthesis of Conformationally Preorganised

Extended $\beta$-Strand Peptidomimetics 